\title{
Optical Design of Camera for Transiting Exoplanet Survey Satellite (TESS)
}

Michael Chrisp, Kristin Clark, Brian Primeau, Michael Dalpiaz, Joseph Lennon

SPIE Conference

\author{
June 7, 2015
}

LINCOLN LABORATORY

244 Wood Street, Lexington MA 02420 


\section{Introduction}

- Design drivers

- Maximum light gathering for size and volume constraints

- Cryogenic operation at $-75^{\circ} \mathrm{C}$

- Wavelength range $600-1000 \mathrm{~nm}$

- Design features

- Seven element hybrid Petzval design

- No vignetting

- Two aspheric surfaces

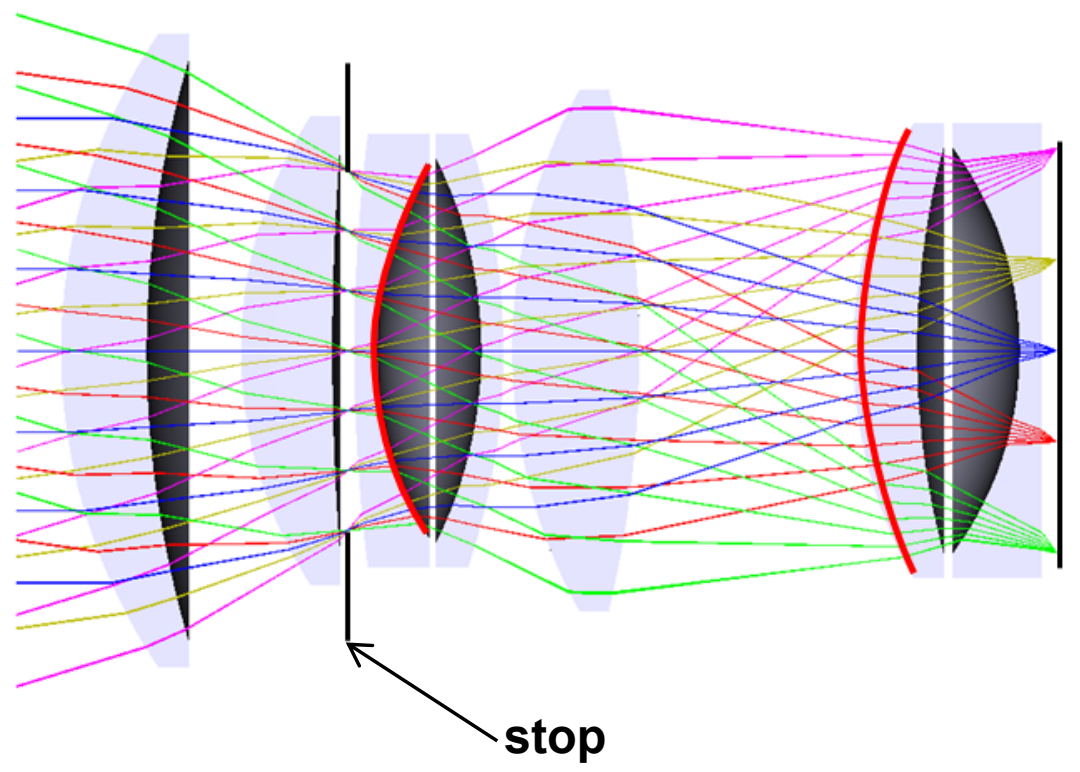

\begin{tabular}{|c|c|}
\hline Parameter & Value \\
\hline FOV & $24^{\circ} \times 24^{\circ}$ \\
\hline FL, f/\# & $146 \mathrm{~mm}, \mathrm{f} / 1.4$ \\
\hline EPD & $105 \mathrm{~mm}$ \\
\hline Wavelengths & $600-1000 \mathrm{~nm}$ \\
\hline CCD & $\begin{array}{l}2 \times 2 \text { detector arrays } \\
4 \mathrm{k} \times 4 \mathrm{k} \text { pixels }\end{array}$ \\
\hline Detector arrays & $\begin{array}{l}2048 \times 2048 \\
15 \text { micron pixels }\end{array}$ \\
\hline Transmittance & $86.5 \%$ (including filter) \\
\hline Mass & $9.3 \mathrm{~kg}$ \\
\hline Dimensions & $\begin{array}{l}17.0 \mathrm{~cm} \text { diameter } \\
21.1 \mathrm{~cm} \text { long }\end{array}$ \\
\hline
\end{tabular}

Optical Design of Camera for TESS - 2 


\section{Design Evolution}

\section{Engineering Design f/1.56}

6 lenses 1 aspheric

Field $22.5^{\circ} \times 22.5^{\circ}$

Pupil $101.8 \mathrm{~mm}$

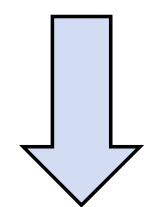

Flight Design f/1.4

7 lenses 2 aspherics

Field $24^{\circ} \times 24^{\circ}$

Pupil 105 mm
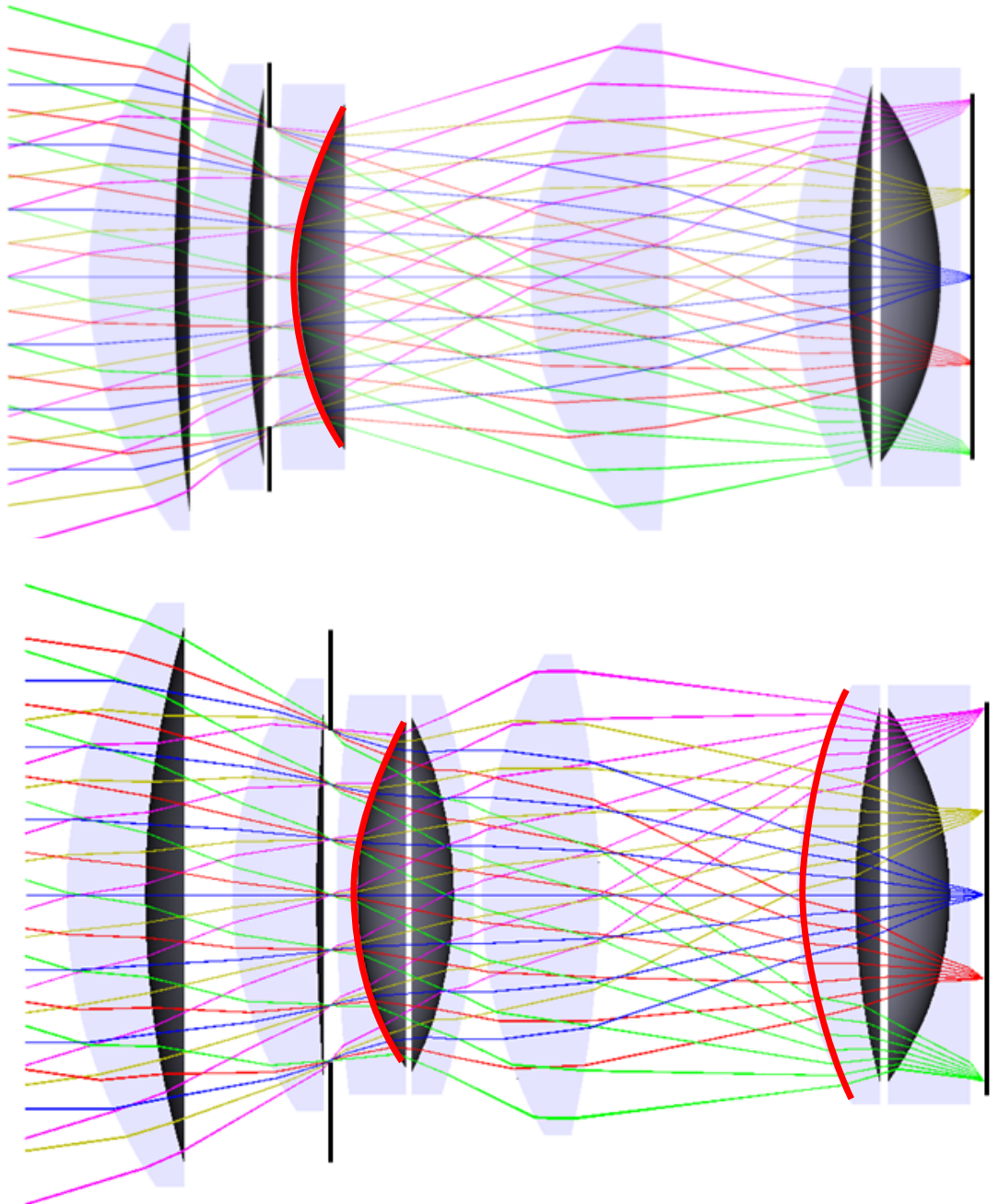


\section{Design Implementation}

Optical Design

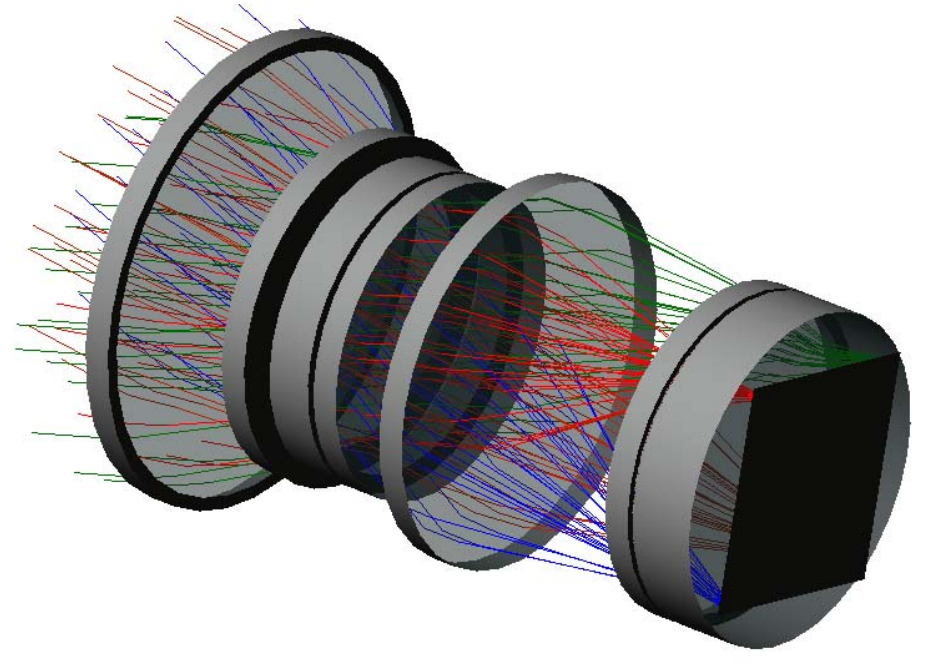

\begin{tabular}{|ll|}
\hline Number of lenses & 7 \\
f-number & 1.4 \\
Pupil diameter & $105 \mathrm{~mm}$ \\
Field of View & $24^{\circ} \times 24^{\circ}$ \\
\hline
\end{tabular}

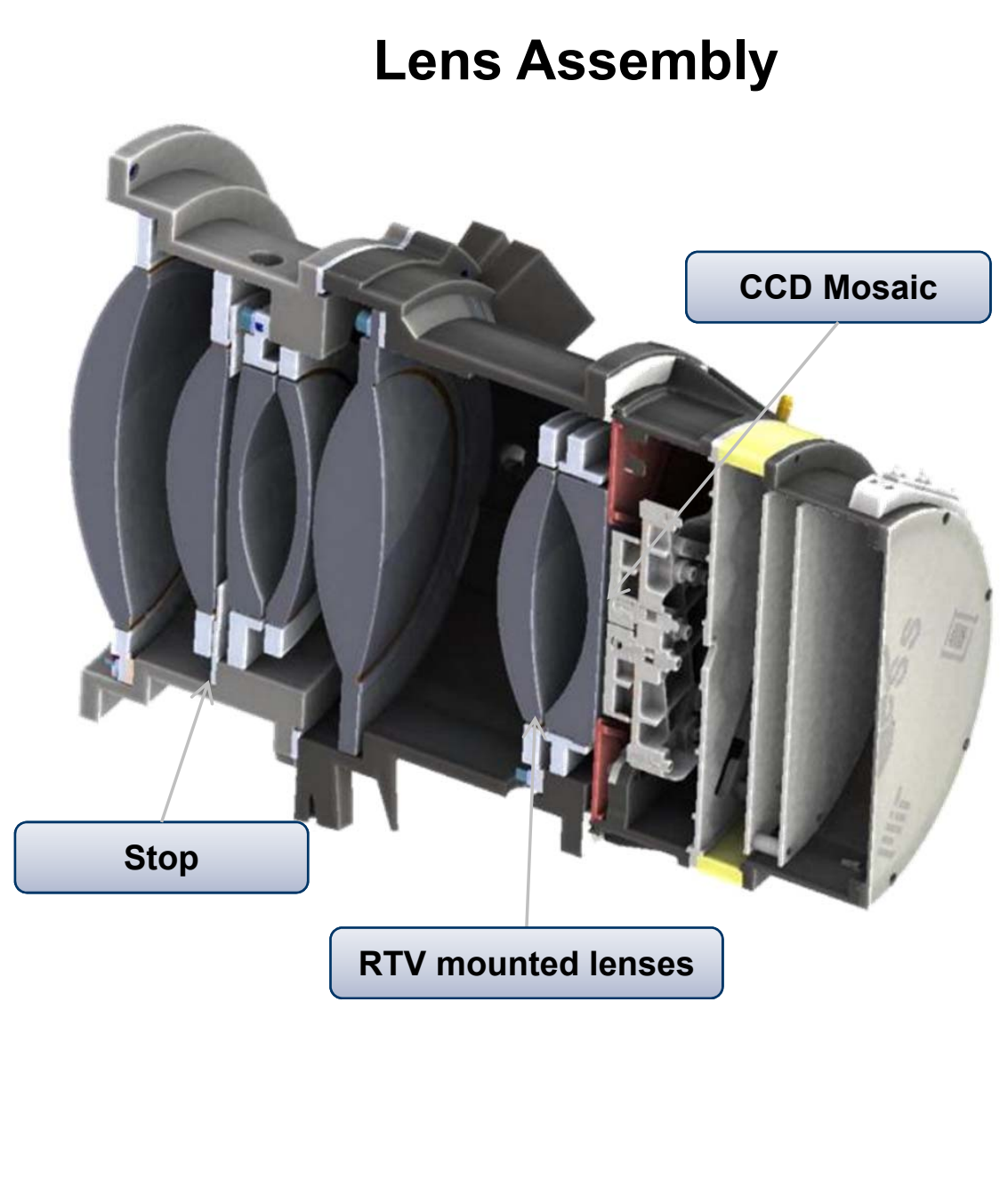




\section{Tolerances}

- Melt data recalculation of design

- Compensators for assembly

- First lens decenter and spacing

- Detector focus

\begin{tabular}{|c|c|c|c|c|c|c|c|c|c|c|}
\hline $\begin{array}{l}\text { L } \\
\text { e } \\
\text { n } \\
\text { s }\end{array}$ & Sur. & $\begin{array}{l}\text { Fringes } \\
\text { (power) }\end{array}$ & $\begin{array}{c}\text { Fringes } \\
\text { (irregular } \\
\text { ity) }\end{array}$ & $\mathbf{d N}$ & $\mathbf{d V}$ & $\begin{array}{c}\text { Lens } \\
\text { wedge } \\
\text { (ETD mm) }\end{array}$ & $\begin{array}{l}\text { Lens } \\
\text { thickness } \\
(\mathrm{mm})\end{array}$ & $\begin{array}{l}\text { Axial } \\
\text { position } \\
\text { (mm) }\end{array}$ & $\begin{array}{l}\text { Radial } \\
\text { decenter } \\
(\mathbf{m m})\end{array}$ & $\begin{array}{l}\text { Lens tilt } \\
\text { (arc min) }\end{array}$ \\
\hline 1 & 1,2 & 3 & 0.5 & \pm 0.00007 & $\pm 0.04 \%$ * & \pm 0.005 & \pm 0.030 & \pm 0.035 & \pm 0.020 & \pm 0.4 \\
\hline 2 & 1,2 & 3 & 0.5 & \pm 0.00007 & $\pm 0.04 \%$ & \pm 0.007 & \pm 0.030 & \pm 0.035 & \pm 0.020 & \pm 0.4 \\
\hline \multirow[t]{2}{*}{3} & 1 & 3 & 0.5 & \pm 0.00007 & $\pm 0.04 \%$ & \pm 0.010 & \pm 0.050 & \pm 0.035 & \pm 0.020 & \pm 0.4 \\
\hline & 2 & 3 & Asp & & & & & & & \\
\hline 4 & 1,2 & 3 & 0.5 & \pm 0.00007 & $\pm 0.04 \%$ & \pm 0.007 & \pm 0.030 & \pm 0.035 & \pm 0.020 & \pm 0.4 \\
\hline 5 & 1,2 & 3 & 0.5 & \pm 0.00007 & $\pm 0.04 \%$ * & \pm 0.007 & \pm 0.030 & \pm 0.035 & \pm 0.020 & \pm 0.4 \\
\hline \multirow[t]{2}{*}{6} & 1 & 3 & Asp & \pm 0.00007 & $\pm 0.04 \%$ & \pm 0.010 & \pm 0.050 & \pm 0.035 & \pm 0.020 & \pm 0.4 \\
\hline & 2 & 3 & 0.5 & & & & & & & \\
\hline 7 & 1,2 & 3 & 1 & \pm 0.00007 & $\pm 0.04 \%$ & \pm 0.007 & \pm 0.025 & \pm 0.035 & \pm 0.020 & \pm 0.4 \\
\hline
\end{tabular}

\begin{tabular}{|l|l|}
\hline ETD - maximum edge thickness minus minimum edge thickness & dN - refractive index difference \\
Fringes power and irregularity - difference from test plate @ 632 nm & dV- Abbe number change
\end{tabular}




\section{Best Aspheric Surface Search}

- Performed search for best aspheric surfaces using Forbes polynomials

- QSL numbers are equivalent to the interferometric fringe density (difficulty of asphere)

- Solution from CODEV Asphere Expert has an RMS Error Value of 45

- $20 \%$ larger RMS spot sizes than the search result and difficult aspheres

- Best solution from search had an RMS error value of 35

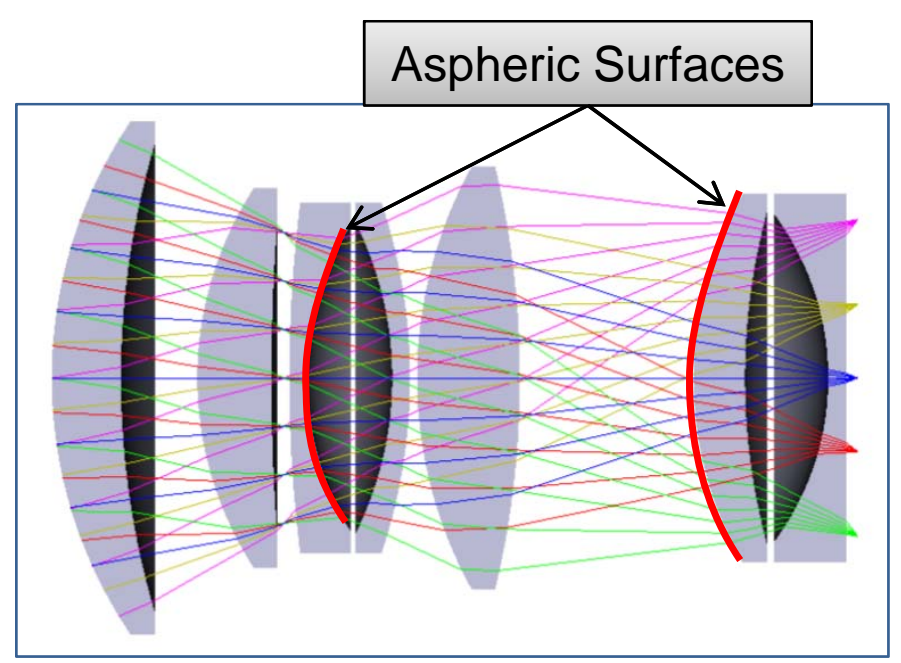

\begin{tabular}{|lccc|}
\hline Aspheric Surfaces & $\begin{array}{c}\text { RMS } \\
\text { Error } \\
\text { Value }\end{array}$ & QSL1 & QSL \\
\hline No aspherics & 97 & NA & NA \\
\hline Single asphere L3, S2 & 59 & 401 & NA \\
\hline $\begin{array}{l}\text { CODEV ASPHERE EXPERT } \\
\text { surface L4, S1 and L5, S1 }\end{array}$ & 45 & 1077 & 1719 \\
\hline Surfaces L3, S2 and L6, S1 & 36 & 451 & 308 \\
\hline
\end{tabular}

The two aspheric surfaces reduce the RMS spot sizes by $60 \%$ compared with the spherical design 


\section{Short Wave Rejection Filter}

- Filter coating on lens saves weight compared with separate filter ahead of lens

- Coating operates over an angle of incidence from $0^{\circ}$ to $30^{\circ}$

- High blocking of $>10^{-5}$ for wavelengths $<530 \mathrm{~nm}$
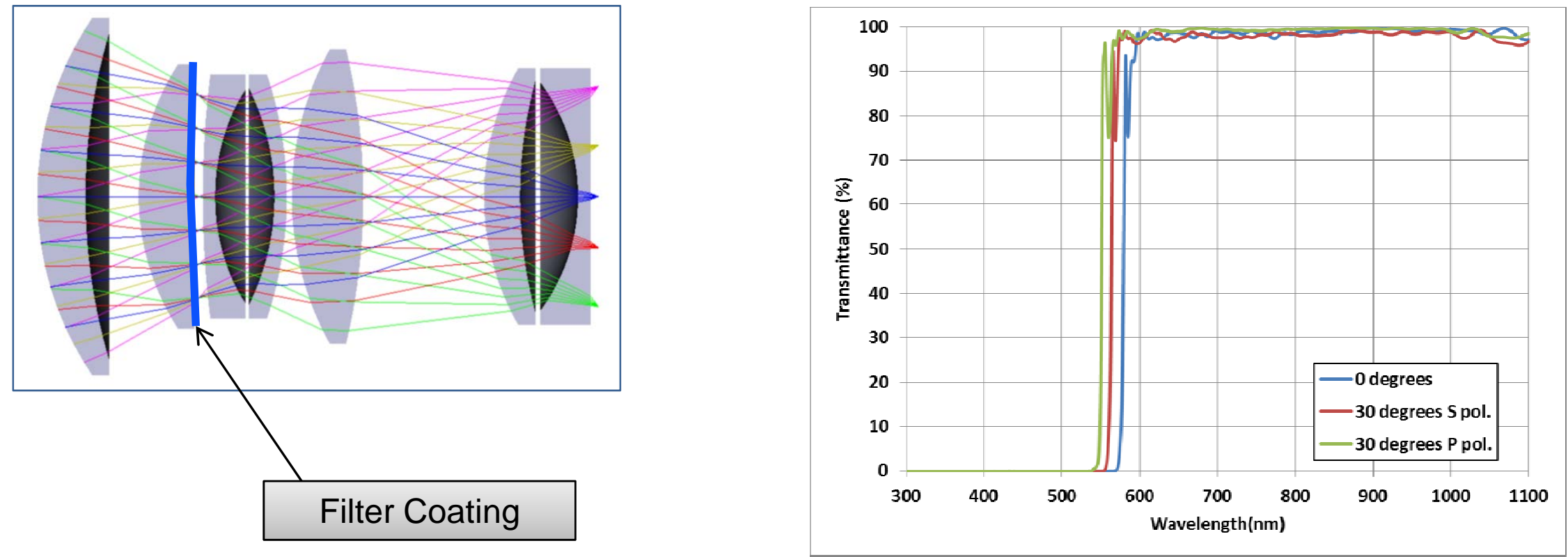


\section{Stray Light}

- Lens hood design blocks Earth- and moonshine from reaching the detector plane

- Two lens hood lengths (required by spacecraft keep-out volume)

- Lens hood model

- Z302 black paint in lens barrel

- IEST-STD-CC1246D level 500A contamination specified on external surfaces

- Baffle achieves $70 \mathrm{~dB}$ isolation at $37^{\circ}$ range for corner fields

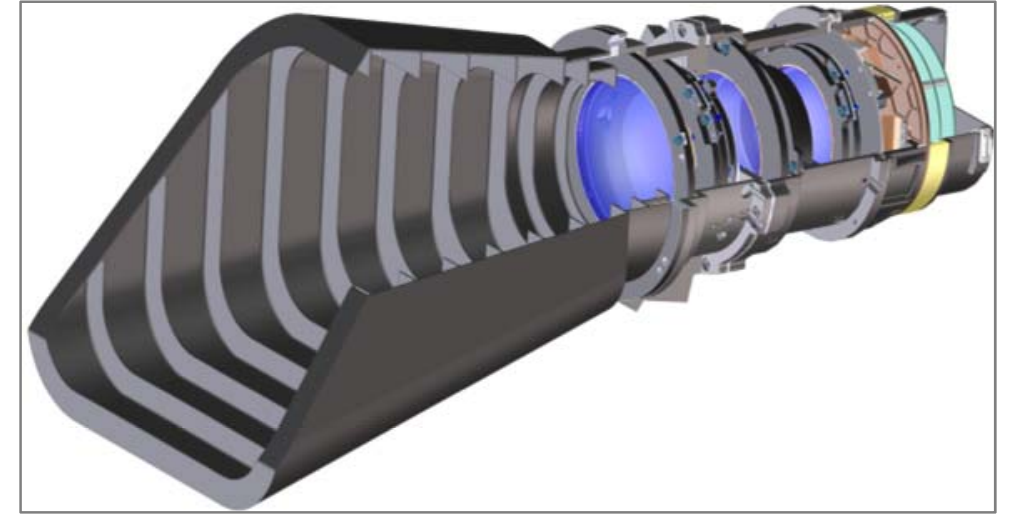

Long Lens Hood

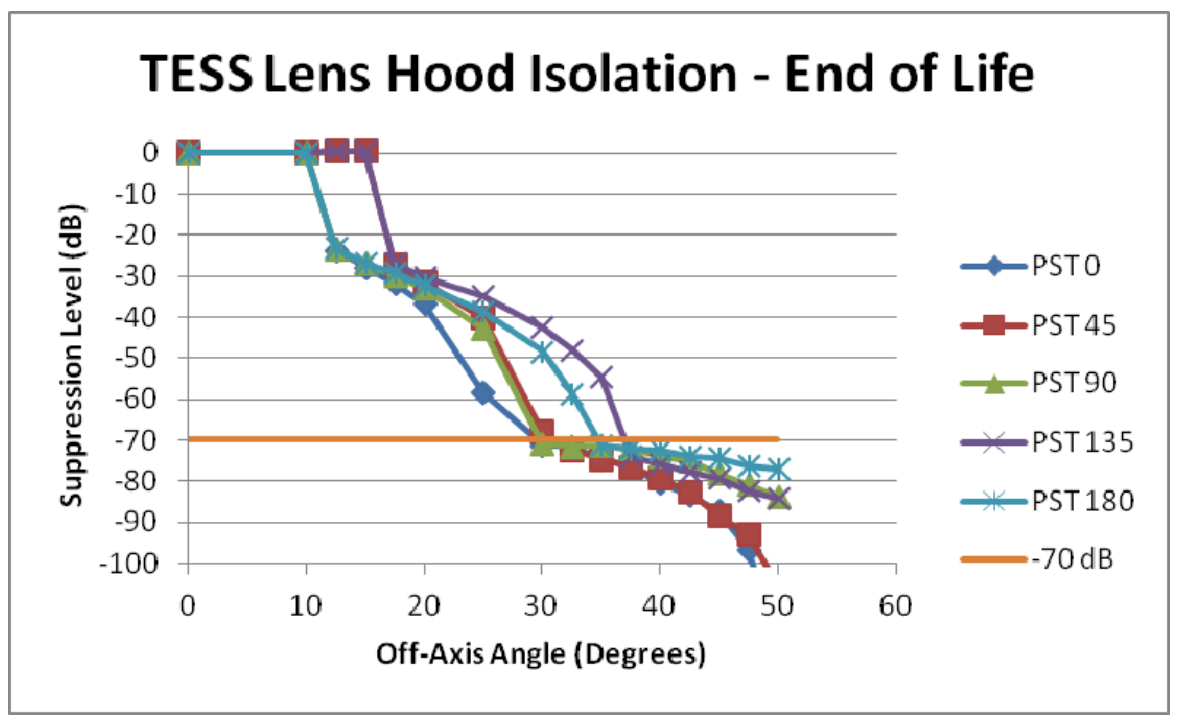




\section{Ghost Image Analysis}

\section{Paraxial ghost analyses showed two paths of interest}

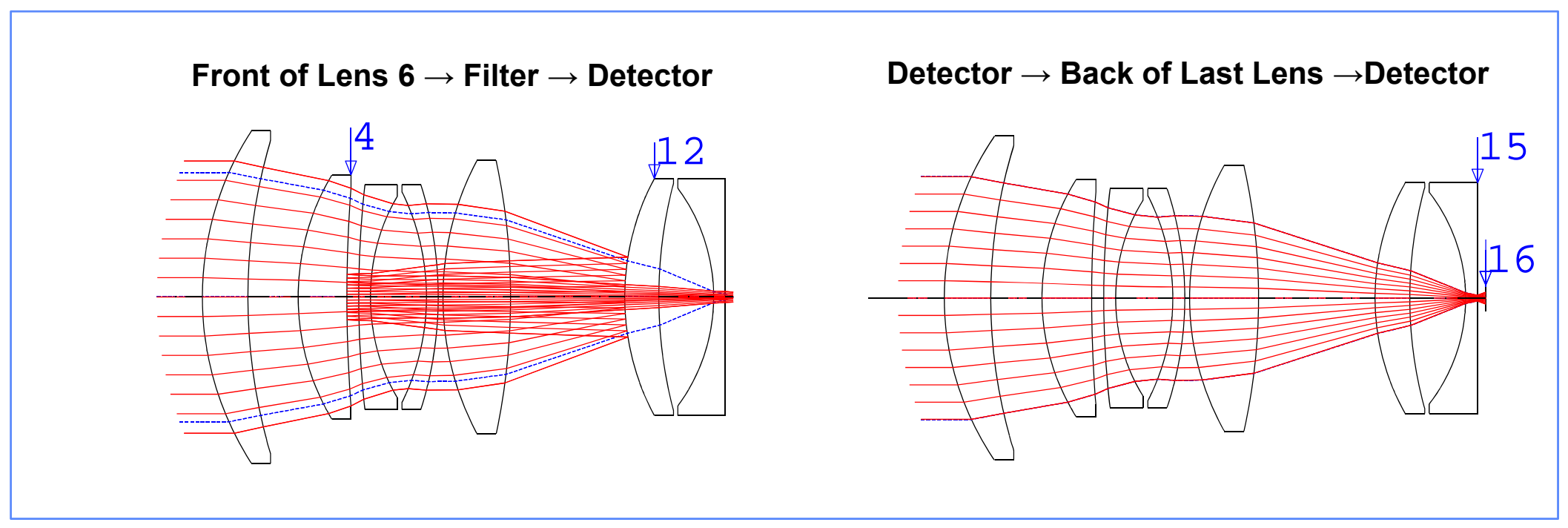

\begin{tabular}{|lcccc|}
\hline \multicolumn{1}{|c}{ Ghost } & $\begin{array}{c}\text { Peak Irradiance } \\
(100 \% \text { transmittance) }\end{array}$ & $\begin{array}{c}\text { Transmittance } \\
\text { Image }\end{array}$ & $\begin{array}{c}\text { Peak Irradiance (with } \\
\text { transmittance) }\end{array}$ & $\begin{array}{c}\text { Ghost Irradiance } \\
\text { relative to Image }\end{array}$ \\
\hline Surfaces $12 \rightarrow 4$ & $4.2 \times 10^{8} \mathrm{~W} \mathrm{~cm}^{-2}$ & 0.84 & $\sim 1.8 \times 10^{8} \mathrm{~W} \mathrm{~cm}^{-2}$ & \\
\hline Surfaces $16 \rightarrow 15$ & $5.6 \times 10^{3} \mathrm{~W} \mathrm{~cm}^{-2}$ & $3.5 \times 10^{-4}$ & $\sim 1.7 \mathrm{~W} \mathrm{~cm}^{-2}$ & $<\sim 10^{-8}$ \\
\hline
\end{tabular}

Ghost images not significant, irradiance $<10^{-8}$ w.r.t. primary image 


\section{Conclusion}

- Hybrid Petzval design with no vignetting proved to have the best light gathering under the volume and weight constrictions

- Two aspheric surfaces improve the r.m.s. spot size by $60 \%$ compared with the non-aspheric design.

- Internal short wave rejection filter coating successfully replaced an external filter

- The lens hood design has met stray light rejection requirements

- Lens has been fabricated and assembled, with initial results matching the performance predictions 